\title{
Recherche médicale: la qualité avant la quantité
}

\section{Le contexte}

Lévidence scientifique constitue un pilier essentiel de la médecine et de sa pratique quotidienne, par exemple dans l'utilisation de directives ou tout au long de la formation (prégraduée, postgraduée et continue). Plusieurs points faibles de la recherche sont connus, notamment les fraudes scientifiques telles que le plagiat ou la manipulation de données ainsi que différentes formes de distorsions (reporting bias, selection bias, etc.) ou de défauts dans la planification, réalisation, évaluation et présentation d'études. Un bon nombre d'entre elles ne sont pas reproductibles et comportent un risque élevé d'interprétation erronée; ceci principalement en raison d'une part de la manière d'évaluer la recherche, à savoir à l'aune du nombre de publications, et de l'autre, de l'influence de l'industrie qui finance des études. Toutes deux créent de faux incitatifs et compromettent la qualité de la recherche. Ces dernières années, le nombre d'études retirées a augmenté et, dans l'opinion publique, la crédibilité de la science est remise en question.

\section{Les arguments}

Les aspects suivants sont essentiels aux yeux de la FMH pour élever et garantir avec efficacité la qualité de la recherche:

\section{Etablir un enregistrement obligatoire}

Différentes analyses montrent que 50\% des études ne sont pas publiées. C'est le cas si les études ne produisent aucun effet, ne confirment pas les connaissances actuelles ou si elles n'obtiennent pas les résultats escomptés. Un enregistrement obligatoire des études

La médecine basée sur les preuves est déterminante pour le quotidien des médecins. Dès lors, une recherche de mauvaise qualité peut aussi avoir un impact sur la sécurité des patients. Pour la FMH, des changements sont nécessaires au niveau du système scientifique pour encourager une recherche de bonne qualité et minimiser les influences négatives. favoriserait la publication complète des résultats, la traçabilité des changements de protocoles et permettrait d'identifier le non-respect des règles et normes scientifiques.

\section{Améliorer la transparence}

Permettre aux experts, rédacteurs de revues spécialisées et autres chercheurs intéressés d'accéder aux protocoles d'études, validations éthiques, données et statistiques améliore la transparence et diminue les risques de fraude scientifique. De plus, les éventuels conflits d'intérêts des chercheurs et le financement des études devraient être communiqués de manière transparente avec la publication ou la présentation des résultats.

\section{Encourager la recherche indépendante}

Les chercheurs ne doivent avoir aucun intérêt pécuniaire ou autre dans les études ou leurs résultats ni participer au marketing de produits. Dans le domaine de la santé, les décideurs doivent intervenir et agir en faveur d'une recherche indépendante de l'industrie. La recherche en médecine doit se focaliser davantage sur les hôpitaux universitaires, les facultés de médecine et les universités et moins sur les poids lourds de l'industrie pharmaceutique.

\section{Système scientifique et évaluation des publications}

La concurrence autour des postes académiques, titres et fonds de recherche met sous pression les chercheurs et les incite à publier vite et beaucoup. En d'autres termes, le système académique mesure une activité de recherche au volume de publications alors que pour une évaluation adéquate de la recherche, il est urgent que la qualité prime de nouveau sur le nombre d'études et de publications. Pour cela, il faut reconnaître et accepter qu'une recherche de qualité élevée a besoin de temps. Seules les études reproductibles et représentatives mènent à des résultats fiables. Lorsqu'une chaire doit être attribuée en fonction d'une activité de recherche, cette dernière doit être évaluée sur la base de la qualité (importance, pertinence, reproductibilité, etc.) et sur la base de publications choisies. Un nombre déterminé de publications ne devrait pas être un critère de décision. 


\section{Vérifier et garantir la qualité des travaux scientifiques}

Dans l'idéal, les instituts de recherche disposent d'un bureau chargé de veiller au respect des règlements et de mettre en place des mesures d'assurance-qualité. Les experts et les rédacteurs de revues scientifiques ont pour mission de vérifier l'enregistrement des études et de consulter ou si nécessaire d'exiger les documents disponibles sur le même sujet d'une étude. Les portails en ligne dédiés à la révision après publication (Post Publication Review) doivent être davantage encouragés, diffusés et utilisés. Enfin, il s'agit de définir des critères clairs et uniformes pour le retrait d'une étude.

\section{Transmettre le savoir et acquérir des compétences}

Avoir une lecture critique de la littérature spécialisée et en évaluer l'évidence présupposent de la méthode et des compétences en la matière. Il s'agit de sensibiliser à long terme à l'importance de la qualité de la recherche. Pour cela, il faut créer une offre institutionnalisée pour la formation prégraduée et postgraduée des médecins et des chercheurs. Les faiseurs d'opinion et les person- nalités exemplaires qui s'engagent en faveur d'une recherche de qualité élevée ont un rôle essentiel à jouer en ce qui concerne la sensibilisation à ces questions.

\section{Nos exigences}

En vue d'encourager la qualité de la recherche, la FMH formule les exigences suivantes:

- La transparence de la recherche doit être améliorée.

- La qualité de la recherche doit être contrôlée au moyen de mesures concrètes.

- Le système d'appréciation académique doit placer la qualité devant le nombre d'études et leur publication.

- Il faut sensibiliser à l'importance de la qualité en matière de recherche et former davantage dans ce domaine.

- Il faut encourager l'indépendance de la recherche présentant des bénéfices pour les patients.

La qualité en médecine. Les médecins s'organisent en faveur de la qualité. 